\title{
Relación entre cohesión y liderazgo en equipos deportivos del departamento de Boyacá - Colombia
}

\author{
Relationship Between Cohesion and Leadership in Sport \\ Teams of the Boyacá's Department, Colombia
}

\section{Relação entre coesão e liderança em equipes esportivos do departamento de Boyacá - Colômbia}

\author{
Erika Losada López, Diana Carolina Rocha y Liliana Castillo \\ Instituto de Deportes de Boyacá \\ Tunja, Boyacá-Colombia
}

\begin{abstract}
Resumen: La presente investigación tiene como objetivo determinar si existe relación entre la cohesión y el liderazgo en los deportistas de las ligas de baloncesto, fútbol sala, fútbol y voleibol del Departamento de Boyacá. Se realiza un muestreo incidental con la participación de 136 jugadores, con edades entre los 13 y 24 años, de género femenino y masculino adscritos al Instituto Departamental de Deportes de Boyacá (Indeportes Boyacá). Investigación de tipo correlacional con diseño transversal, utilizando el Instrumento Multidimensional de Cohesión en el Deporte IMCODE (Multidimensional Sport Cohesion Instrument, MSCI) y la Escala de Evaluación del Liderazgo Deportivo (EELD), validados para la población objeto de estudio. Los resultados evidencian una relación entre las dimensiones calidad de trabajo-aspectos relativos a la ejecución (cohesión) y valores deportivos (liderazgo).

Palabras clave: Cohesión, Equipo, Liderazgo, Psicología del deporte.

Abstract: The present investigation has as objective to determine if exists relationship between the cohesion and the leadership in the sportsmen of the leagues of basketball, futsal, football and volleyball of the Department of Boyacá. They are carried out an incidental sampling with the participation of 136 players, with ages between 13 and 24 years, of feminine and masculine gender attributed to the Departmental Institute of Sports of Boyacá (Indeportes Boyacá). Investigation of type correlational with traversal
\end{abstract}

design, using the Multidimensional Instrument of Cohesion in the Sport IMCODE (Multidimensional Sport Cohesion Instrument, MSCI) and the Scale of Evaluation the Sport Leadership (EELD), validated for the population study object. The results evidence a relationship among the dimensions quality of relative work-aspects to the execution (cohesion) and sport values (leadership).

Key words: Cohesion, Leadership, Sports Psychology and Team.

Resumo: A presente pesquisa tem como objetivo determinar se existe relação entre a coesão e a liderança nos esportistas das ligas de basquete, futebol sala, futebol e voleibol do Departamento de Boyacá. Realiza uma mostra incidental com a participação de 136 jogadores, com idades entre 13 e 24 anos, do gênero masculino e feminino, inscritos no Instituto Departamental de Deportes de Boyacá (Indeportes Boyacá). A pesquisa do tipo correlacional com desenho transversal, utilizando o Instrumento Multidimensional de Coesão no Esporte IMCODE (Multidimensional Sport Cohesion Instrument, MSCI) e a escala de Avaliaçấo da Liderança Esportiva (EELD), validados pela população objeto do estudo. Os resultados evidenciaram uma relaçâo entre as dimensōes de qualidade de trabalho - aspectos relativos a execução (coesão) e os valores esportivos (liderança).

Palavras Chave: coesão, equipe, liderança, psicologia do esporte.

\section{Introducción}

En los últimos años la aplicación de la psicología al ámbito deportivo ha experimentado un gran auge debido a los beneficios que esta ciencia aporta al contexto deportivo (A. García, 2009); es así como a través de la preparación psicológica que se realiza tanto en deportes individuales como en equipo se mejoran destrezas psicológicas necesarias para la práctica del deporte, tales como; la motivación, ansiedad, emoción, agresión, actitudes, hábitos alimenticios, atención, percepción, concentración, entre otras. Sin embargo, en deportes de equipo, destrezas como la comunicación, cohesión, liderazgo y cooperación son determinantes permitiendo realizar intervenciones psicológicas que benefician al equipo, con el fin de conseguir los objetivos planteados por éste y lograr al tiempo la consolidación del mismo.

En este punto es importante hacer una clara diferenciación entre los términos equipo y grupo deportivo. El primero, hace referencia a un conjunto de personas interrelacionadas con identidad que se organizan para llevar a cabo una tarea determinada, en cada uno de sus miembros existe una necesidad del "otro", es decir, existe un sentido de interdependencia entre sus integrantes y como consecuencia de estas características cada uno de ellos posee y mantiene un rol que complementa al resto del equipo. Por otro lado, el grupo deportivo se refiere a ese conjunto de personas sin considerar la tarea para 
la que han formado un conjunto, considerándolo en su totalidad, es decir, no existe una tarea específica lo que impide que se realice una asignación concreta de los roles ("Diferencia equipo - grupo," 2009).

Por otra parte, el equipo deportivo ha sido definido recientemente por García-Mas, Olmedilla, Morilla, Rivas, García y Ortega (2006), como un conjunto de personas que trabajan para realizar una tarea, comparten responsabilidades en cuanto a los resultados colectivos y se ven a sí mismos y por los demás como entidades sociales. Desde esta conceptualización se evidencia una dinámica de grupo desde la cual, cada uno de los miembros del equipo es responsable de dirigir e involucrar su rol o trabajo hacia la consecución del objetivo pensando en la obtención del beneficio colectivo, más no, en el individual.

En los equipos deportivos se deben establecer normas para evitar dificultades. Carron (1991; citado por Ortín, 2002), la define como una pauta de conducta, cuyo cumplimiento es necesario para descartar la posibilidad de que las responsabilidades por el incumplimiento recaigan sobre un solo miembro (e.g. el cuerpo técnico); permite que se desarrolle de manera más adecuada la cohesión incrementando a su vez el nivel de acatamiento de las normas.

Teniendo en cuenta lo anterior, y la importancia que tiene la cohesión en el funcionamiento óptimo del equipo, es necesario hacer una conceptualización de este término. Los primeros acercamientos teóricos abordan la cohesión deportiva como un constructo unidimensional enfocado principalmente a los aspectos sociales. Para Spalinsky (1978, en Sabas y Del Pino, 2009), "la cohesión corresponde a una característica integral de los vínculos dentro del grupo que muestra el nivel de coincidencia de opiniones, valoraciones y posiciones de los miembros del mismo; en lo referente a los objetivos que le son importantes" (p. 26); posteriormente surgió una de las conceptualizaciones más consolidadas, la de Carron (1982; citado por Dosil, 2004) el cual la define como "un proceso dinámico que se refleja en la tendencia de un grupo a no separarse y a permanecer unido en la búsqueda de sus metas y objetivos" (p. 248); esta definición hace referencia a dos dimensiones: la cohesión de tarea que refleja el grado en que los miembros del grupo trabajan juntos para alcanzar objetivos comunes y la cohesión social que son los lazos de atracción interpersonal que unen a los miembros del grupo (Carron, Widmeyer, Brawley, 1985; Carron, 1998; citados por Leo, García, Sánchez y Parejo, 2008).

De esta manera la cohesión social es lo que lleva a desarrollar y mantener las relaciones sociales dentro del equipo; mientras que la cohesión de tarea está dada por el grado de implicación del equipo con el logro del objetivo (Carless y De Paola, 2000; citados por Picazo, Zornoza y Peiró, 2009). De acuerdo con lo anterior, la participación social y la centrada en la tarea contribuyen a la cohesión en los equipos, aunque la participación en la tarea es la más visible en la etapa de formación del grupo, acordando tareas y objetivos; una vez establecidos, sus miembros pueden dedicar más tiempo a la interacción social familiarizándose unos con otros (Carron y Brawley, 2000 citados por Picazo et al. 2009).

Debido al interés que ha despertado este proceso en la Psicología del Deporte se han creado diversos modelos que han intentado dar explicación a esta dinámica. El modelo de cohesión en el deporte más reconocido y utilizado es el desarrollado por Carron, el cual menciona cuatro antecedentes principales que afectan el desarrollo de la cohesión: a) Factores ambientales o las fuerzas normativas que mantienen juntos a los miembros de un equipo; incluye las regulaciones contractuales, las reglas de reclutamiento, las normas de convivencia; b) factores personales o características individuales, sus condiciones físicas, técnicas y psicológicas, su experiencia e historia deportiva; c) factores de liderazgo, el estilo y los comportamientos del entrenador en interacción con el equipo y d) factores del equipo, características de tarea del equipo, normas de productividad, formas de relación, actividades a realizar y estabilidad de sus miembros, etc., (Carron, 1982 citado por Velásquez, 2005).

En esta misma línea, es importante conocer e identificar relaciones entre cohesión y rendimiento; teóricamente existe un dilema en cuanto a la dirección de causalidad entre estas dos variables; para algunos autores (Grieve y cols, 2000 citado por Díez y Márquez, 2005) es el rendimiento el que influye positivamente en la consecución o consolidación de la cohesión de equipo; así, el impacto del rendimiento sobre la cohesión llega a ser más importante encontrándose la cohesión más relacionada con la satisfacción, la conformidad y la estabilidad que con el rendimiento propiamente dicho. En la otra dirección es la cohesión la que influye positivamente en el rendimiento deportivo (González, 2007).

Algunos estudios, como los mencionados por Salazar (2006, citando a Martens y Peterson, 1971), relacionan la cohesión con el rendimiento deportivo, determinando que los equipos que muestran un alto grado de comunicación, solidaridad y metas comunes, tienen una mayor probabilidad de alcanzar el éxito grupal y un mejor rendimiento. Así mismo, Briones y Tabernero (2005) concluyen, que cuando el grupo se forma en aspectos cooperativos y de aprendizaje, resultan favorecidos procesos como la comunicación abierta y la cohesión social. En la investigación realizada por Carron y Dennis, (2001; citados por Balaguer, Castillo, Moreno y Soriano, 2004) se concluye que, la cohesión proporciona mayor aprendizaje, productividad, comunicación, satisfacción con el deporte y con los compańeros, sentimientos de seguridad y adherencia al deporte.

Para obtener el éxito deportivo, los deportistas deben comprender y aceptar su rol dentro del equipo; así, como aceptar al mismo tiempo el de sus compañeros, ser capaz de solucio- 
nar los conflictos que surjan al interior del mismo de una forma racional, cumplir las normas establecidas, prestar ayuda y/o apoyo constante a sus compañeros, establecer líneas de comunicación con el entrenador y con los demás miembros y preocuparse por superarse cada vez no sólo como deportista sino como persona (Dosil, 2004).

González (2007), indica que el trabajo de construir un equipo requiere de la acción de todos los miembros del grupo, pero que es necesario el protagonismo de diferentes personas con gran poder de influencia; como es el caso del entrenador y los capitanes. De esta forma, se reconoce la importancia del liderazgo, considerándolo como aspecto clave para el funcionamiento de los equipos deportivos.

En esta línea Barrow, (1977; citado por Hardy y Jones, 1996), señala que desde el ámbito del deporte se cree que el rol del líder corresponde a un atributo natural del entrenador, el instructor o el capitán de un equipo; sin embargo, la condición de liderazgo no es simplemente una característica personal; más bien, puede considerarse como un tipo de relación social compleja que engloba un proceso conductual en un equipo en función de sus objetivos. Por lo tanto, existe un liderazgo informal, donde algunos de sus miembros influyen de manera permanente; esta relación social no puede ser entendida sólo a partir del líder, sino que se extiende a sus seguidores y a las situaciones que de manera programada o espontánea se presentan en dicha relación, como la precisión del tiempo, las exigencias de un torneo, el nivel de competencia del equipo, etc.

Arce, Torrado, Andrade, Garrido y De Francisco (2008), plantean la importancia de diferenciar líder formal del informal. Un líder formal, se caracteriza por poseer y practicar algún tipo de poder sobre los miembros del equipo, siendo generalmente este impuesto por las directivas del club deportivo (e.g. entrenador), situación que lo lleva a ejercer un liderazgo diferente al que ejercería un miembro del equipo (deportista) al cual sus compañeros le han atribuido informalmente el rol de líder por características como el compañerismo, colaboración, vocería del equipo, etc.; así, teniendo en cuenta estas particularidades generalmente el líder informal será el que tendrá mayor influencia sobre el grupo debido a la cercanía de este con los demás miembros del equipo.

Davies (1991; citado por Ruiz, 2006), señala como características de los lideres en los equipos deportivos las siguientes particularidades de deportistas de éxito que llegan a convertirse en lideres potenciales de sus equipos: a) disfrute de las relaciones sociales; b) generosidad en la valoración y relación con los demás; c) capacidad y deseo real de ayudar; d) deseo de desarrollar relaciones interpersonales estimulantes, cálidas y seguras consideradas como de vital importancia para el desarrollo de la motivación y habilidad de los deportistas; e) entusiasmo y creatividad; f) buena organización y capacidad para ser consciente; g) motivación por el entrenamiento y respeto por sus compañeros; h) persona estable y generador de confianza; i) compromiso; j) conocimiento de sus compañeros; y k) importancia de su liderazgo; de este modo un deportista que pueda reunir estas características a lo largo de su desarrollo deportivo es muy probable que logre destacarse entre los demás miembros de su equipo.

Los estudios de liderazgo en el deporte, se han orientado a analizar el liderazgo del entrenador (Chelladurai y Saleh, 1980; Martens, 1987; Smoll, et al., 1977; citados por García, 2004), dejando de lado el rol del deportista como líder; pero sin lugar a dudas estudios como los de Loughead y Hardy (2005; citados por Arce et al. 2008) han contribuido a orientar el análisis a la diferencia entre los comportamientos de ambos tipos de líderes en los equipos deportivos mostrando que, mientras los entrenadores exhiben comportamientos de carácter más autoritario y más orientados a la tarea; los jugadores líderes tienden a mostrar más comportamientos democráticos, de apoyo social, orientados a las relaciones y al refuerzo positivo, obteniendo en el campo de juego mayor aceptación los segundos que los primeros.

Arce et al. (2008), señalan algunas investigaciones que han abordado la figura del jugador líder de manera específica analizando sus características, dimensiones y su relación con otras variables, con el objetivo de describir e identificar a los sujetos que ejercen ese liderazgo; tal es el caso de los trabajos de Rees y Segal (1984), desde los cuales se profundiza acerca de los estilos de liderazgo en equipos deportivos, recogiendo la tradicional idea que distingue entre el líder orientado a la tarea, rol o meta y el orientado a lo social mostrando una afinidad entre los miembros de un grupo; Glenn y Horn (1993), señalan diferencias significativas en la relación entre el nivel de juego y la capacidad de liderazgo, mostrando mayores puntuaciones en liderazgo, los deportistas con un alto nivel de juego; finalmente con relación a la posición en el campo de juego los estudios de Klonsky, (1991); y Lee, Coburn y Partridge, (1983); concluyen que los jugadores que se sitúan en posiciones centrales poseen altos niveles de liderazgo dentro de su equipo.

Velásquez (2005), hace referencia a la investigación sobre el liderazgo llevada a cabo en la década de los años 80, focalizada en un inicio en descubrir y resaltar las características personales de los líderes exitosos en el terreno de juego; posteriormente, las investigaciones se dirigieron a estudiar la caracterización de los rasgos de la personalidad del líder, ignorando las interacciones con sus compañeros de grupo y los factores situacionales que influían o modificaban dicha relación. En años recientes el interés en el tema ha ido cambiando gradualmente hacia la construcción y aplicabilidad de modelos interactivos, que consideren la relativa afectividad de los estilos de liderazgo que se pueden ejercer dentro de las múltiples y diversas situaciones de juego de las disciplinas deportivas. 
Para Dosil (2004), al trabajar el desarrollo de habilidades del liderazgo en el deporte se debe tener en cuenta que existen varios tipos de liderazgo, entre los cuales los más usuales son el liderazgo del directivo, del entrenador y del jugador; así cualquier tipo de liderazgo, para ser efectivo debe presentar un equilibrio entre: las cualidades del líder, estilos de liderazgo, factores de la situación y cualidades del seguidor; de este modo se busca que dicho líder se convierta en un líder efectivo a través del asesoramiento en la toma de decisiones efectivas, mejora de los estilos de comunicación (verbal y no verbal), análisis de situaciones, etc., ya sea adquiriendo o perfeccionando estas habilidades y contribuyendo, así a la mejora del equipo.

\section{Método}

\section{Participantes}

La muestra la conformaron un total de 136 deportistas, pertenecientes a Indeportes Boyacá - Colombia, los cuales fueron seleccionados de manera incidental según los siguientes criterios de inclusión: Ser miembros de las ligas de baloncesto, fútbol sala, fútbol y voleibol del Instituto, hombres y mujeres entre los 13 y 24 años de edad, con participación en competencias durante el último año y que asistan a entrenamientos de su deporte mínimo cuatro de los siete días a la semana.

\section{Instrumentos}

Para la medición de las variables objeto de estudio de esta investigación se hace uso de los siguientes instrumentos:

- Instrumento Multidimensional de Cohesión en Deporte (IMCODE): Adaptación espańola del Multidimensional Sport Cohesion Instrument (MSCI) realizada por García, Rodríguez, Andrade, y Arce, (2006) compuesta por 22 ítems distribuidos en cuatro factores: Atracción hacia el grupo, Calidad de trabajo-relaciones interpersonales, Calidad de trabajo-aspectos relativos a la ejecución y Roles valorados. Es un instrumento de lápiz y papel, las respuesta al mismo están en una escala tipo Likert de 10 puntos ( 0 a 10$)$ y etiquetas al lado de estos valores mínimo y máximo de la escala, la aplicación es individual o colectiva y con un tiempo variable entre 20 y 30 minutos. Este instrumento ofrece una validez satisfactoria con un Alpha de Cronbach entre 0.845 y 0.5609 para los factores medidos por el instrumento.

- Escala de Evaluación del Liderazgo Deportivo: (EELD) Arce, Torrado, Andrade, Garrido y De Francisco, (2008): Está diseńada para medir el liderazgo deportivo en deportistas por medio de 30 ítems, divididos en dos grandes dimensiones: Orientación social compuesta por cuatro sub-dimensiones: empatía, influencia en la toma de decisiones, valores deportivos y apoyo social y Orientación a la tarea. El objetivo de esta escala es determinar la capacidad de liderazgo de los deportistas sobre los demás miembros del equipo y estipular cuales pueden ejercer esta función entre sus compañeros. Instrumento de lápiz y papel, con 5 opciones de respuesta según escala tipo likert donde 1 corresponde a casi nunca, como valor mínimo y 5 casi siempre, como valor máximo; se aplica individual o colectivamente y tiene una duración aproximada de 20 minutos. Esta escala muestra una fiabilidad satisfactoria con un Alpha de Cronbach entre 0,849 y 0,776 para los factores medidos.

\section{Procedimiento}

Debido a la inexistencia de instrumentos que evalúen estos procesos, cohesión y liderazgo deportivo en Colombia, se realiza una adaptación; con una validez de contenido, por dos Psicólogos Especialistas en Psicología del Deporte y el Ejercicio Físico, egresados de la Universidad el Bosque; para establecer su consistencia interna se utiliza el índice kappa de Cohen donde se cuantifica el grado de acuerdo entre dos observadores denominado fiabilidad. Luego se realiza una validez de criterio con un pilotaje a 44 deportistas (22 mujeres y 22 hombres) que cumplieran los mismos criterios de inclusión que los participantes objeto de estudio con el coeficiente de fiabilidad Alpha de Cronbach midiendo la consistencia interna y determinando la coherencia de los instrumentos.

Los resultados indican un índice de coeficiente de fiabilidad Kappa de Cohen, entre 0.994 y 0.999 para los criterios de pertinencia, suficiencia y ubicación para la EELD. Para el IMCODE el índice de fiabilidad es de 1 para el criterio de pertinencia siendo perfecto; para el criterio de suficiencia se obtiene un índice significativo de 0.999 y para el criterio de ubicación 0.977; es decir, se predice algún grado de concordancia entre los jueces, corrigiendo de esta manera el factor azar, determinando que estos instrumentos poseen una muy buena fiabilidad en concordancia entre los observadores (Validación de instrumentos, 2009). Las puntuaciones bajas estuvieron presentes en el criterio de claridad (EELD 0.3456; IMCODE 0.038), lo que indica la necesidad de realizar ajustes a los instrumentos.

Para la validez de criterio se señala un índice de fiabilidad Alpha de Crombach de 0.98 siendo una correlación positiva muy alta para la EELD y el IMCODE muestra un índice de correlación positivo alto de 0.77 ; siendo ambas correlaciones muy significativas, lo que indica una correlación positiva alta, es decir, alta fiabilidad; entre más heterogéneo es el grupo de deportistas mayor es el coeficiente de confiabilidad. Este 
coeficiente no es una propiedad exclusiva del instrumento en sí, sino que es una propiedad del instrumento para un determinado grupo de personas en una determinada situación (Silveira, 1993; citado por Validación de instrumentos, 2009).

Posteriormente se seleccionan los deportistas que cumplen los criterios de inclusión; a los cuales se les brinda la información necesaria dando a conocer el objetivo de la investigación, por medio del uso del consentimiento informado teniendo en cuenta las consideraciones éticas, garantizando la total y plena confidencialidad de los datos recolectados.

Se realiza la aplicación de los instrumentos de manera colectiva y se procede a analizar la información recolectada por medio de un análisis de correlación lineal con el estadísti- co de Pearson de los cuatro factores del IMCODE y de los cinco factores del EELD, utilizando el paquete estadístico SPSS versión 17; determinando la relación existente entre la cohesión y el liderazgo de los equipos deportivos (baloncesto, fútbol sala, fútbol y voleibol) de Indeportes Boyacá.

\section{Resultados}

Se presentan los estadísticos descriptivos (total, media, desviación estándar e intervalo de confianza) de forma general, sobre las percepciones de cohesión y liderazgo de los deportistas de las ligas de baloncesto, futbol, futbol sala y voleibol, tal y como se observa en la Tabla 1 .

Tabla 1. Estadisticos descriptivos

\begin{tabular}{|c|c|c|c|c|c|}
\hline Variables & Total & $M$ & $S$ & D.S & I. $C$ \\
\hline \multicolumn{6}{|l|}{ Cohesión } \\
\hline Calidad de trabajo (ejecución) & 10137,0 & 74,54 & 111,6 & 10,6 & $69,5-83,0$ \\
\hline Atracción hacia el grupo & 7504,0 & 55,17 & 27,70 & 5,26 & $53,0-59,0$ \\
\hline Roles valorados & 4509,0 & 33,15 & 32,91 & 5,73 & $30,0-40,0$ \\
\hline Calidad de trabajo (interpersonales) & 2548,0 & 18,73 & 32,12 & 5,66 & $14,0-30,0$ \\
\hline \multicolumn{6}{|l|}{ Liderazgo } \\
\hline Empatía & 2856,0 & 21,00 & 6,24 & 2,49 & $19,6-25,9$ \\
\hline Orientación a la Tarea & 3196,6 & 23,50 & 10,08 & 3,17 & $21,6-29,1$ \\
\hline Influencia en la toma de decisiones & 2858,1 & 21,01 & 7,68 & 2,77 & $19,6-27,5$ \\
\hline Valores deportivos & 3136,4 & 23,06 & 7,67 & 2,77 & $21,4-30,0$ \\
\hline Apoyo social & 2573,8 & 18,92 & 5,97 & 2,44 & $17,5-23,9$ \\
\hline
\end{tabular}

\section{Total EELD 145}

Total IMCODE 220

Los resultados indican que con relación a la variable cohesión, la escala que obtiene una mayor puntuación es Calidad de trabajo-aspectos relativos a la ejecución ( $\mu=74,54$; $\mathrm{DS}=10.6)$ y la escala con la puntuación inferior es Calidad de trabajo-relaciones interpersonales. En la variable liderazgo la escala que reporto una mayor calificación es orientación a la tarea $(\mu=23,50, \mathrm{DS}=3.17)$, por el contrario apoyo social obtuvo una baja calificación $(\mu=18,92, \mathrm{DS}=2.44)$.

La tabla 2 muestra los estadísticos descriptivos por deporte, en cuanto a la variable cohesión, se observa que el deporte fútbol sala obtiene una mayor puntuación comparado con los demás equipos en la dimensión de roles valorados $(\mu=196,03$; $\mathrm{DS}=107.30$ ). Baloncesto otorga una puntuación mayor a la escala de calidad de trabajo-aspectos relativo a la ejecución $(\mu=69,29, \mathrm{DS}=7.66)$, al igual que para fútbol $(\mu=77,92$, $\mathrm{DS}=10.47)$ y voleibol $(\mu=71,71, \mathrm{DS}=7.85)$. La puntuación de menor calificación para baloncesto $(\mu=19,51, \mathrm{DS}=5.52)$, fútbol $(\mu=19,03, \mathrm{DS}=5.94)$, voleibol $(\mu=17,53, \mathrm{DS}=4.58) \mathrm{y}$ fútbol sala $(\mu=18,77, \mathrm{DS}=6.611)$ corresponde a la escala de calidad de trabajo-relaciones interpersonales. 
Tabla 2. Estadísticos descriptivos por deporte

\begin{tabular}{|c|c|c|c|c|c|c|}
\hline Variables & Total & $M$ & $S$ & D.S & I.C & \\
\hline \multirow{11}{*}{ Baloncesto } & Cohesión & & & & & \\
\hline & Calidad de trabajo (ejecución) & 1871,0 & 69,29 & 58,75 & 7,66 & $65,0-73,0$ \\
\hline & Atracción hacia el grupo & 1459,0 & 54,03 & 44,19 & 6,64 & $50,0-59,0$ \\
\hline & Roles valorados & 852,0 & 31,55 & 25,64 & 5,06 & $27,0-36,0$ \\
\hline & Calidad de trabajo (interpersonales) & 527,0 & 19,51 & 30,49 & 5,52 & $14,0-26,0$ \\
\hline & Liderazgo & & & & & \\
\hline & Empatía & 528,6 & 19,57 & 5,90 & 2,43 & $17,1-21,8$ \\
\hline & Orientación a la Tarea & 585,4 & 21,68 & 16,88 & 4,10 & $17,3-25,7$ \\
\hline & Influencia en la toma decisiones & 527,4 & 19,53 & 17,57 & 4,19 & $15,6-23,2$ \\
\hline & Valores deportivos & 599,9 & 22,21 & 6,46 & 2,54 & $19,7-24,2$ \\
\hline & Apoyo social & 488,9 & 18,10 & 5,14 & 2,26 & $16,0-20,2$ \\
\hline \multirow{11}{*}{ Fútbol } & Cohesión & & & & & \\
\hline & $\begin{array}{l}\text { Calidad de trabajo } \\
\text { (ejecución) }\end{array}$ & 4286,0 & 77,92 & 109,69 & 10,47 & $74,0-85,0$ \\
\hline & Atracción hacia el grupo & 3057,0 & 55,58 & 22,17 & 4,70 & $54,0-59,0$ \\
\hline & Roles valorados & 1920,0 & 34,90 & 29,71 & 5,45 & $33,0-39,0$ \\
\hline & Calidad de trabajo (interpersonales) & 1047,0 & 19,03 & 35,29 & 5,94 & $15,0-24,0$ \\
\hline & Liderazgo & & & & & \\
\hline & Empatía & 1153,2 & 20,96 & 7,41 & 2,72 & $19,6-23,0$ \\
\hline & Orientación a la Tarea & 1346,2 & 24,47 & 7,25 & 2,69 & $23,0-29,0$ \\
\hline & Influencia en la toma decisiones & 1163,1 & 21,14 & 5,35 & 2,31 & $20,0-22,8$ \\
\hline & Valores deportivos & 1278,6 & 23,14 & 10,19 & 3,19 & $22,0-25,0$ \\
\hline & Apoyo social & 1035,2 & 18,82 & 7,34 & 2,71 & $18,0-20,0$ \\
\hline \multirow{11}{*}{ Voleibol } & Cohesión & & & & & \\
\hline & Calidad de trabajo (ejecución) & 2295,0 & 71,71 & 61,75 & 7,85 & $69,0-76,0$ \\
\hline & Atracción hacia el grupo & 1780,0 & 55,62 & 16,11 & 4,01 & $54,0-59,0$ \\
\hline & Roles valorados & 982,0 & 30,68 & 27,89 & 5,28 & $26,0-34,5$ \\
\hline & Calidad de trabajo (interpersonales) & 561,0 & 17,53 & 21,03 & 4,58 & $14,0-21,0$ \\
\hline & Liderazgo & & & & & \\
\hline & Empatía & 698,6 & 21,83 & 5,54 & 2,35 & $20,2-23,7$ \\
\hline & Orientación a la Tarea & 759,6 & 23,73 & 9,96 & 3,15 & $20,9-26,6$ \\
\hline & Influencia en la toma decisiones & 686,7 & 21,46 & 5,45 & 2,33 & $20,0-22,8$ \\
\hline & Valores deportivos & 747,6 & 23,36 & 8,00 & 2,82 & $21,0-25,7$ \\
\hline & Apoyo social & 623,2 & 19,47 & 4,47 & 2,11 & $17,8-21,1$ \\
\hline
\end{tabular}




\begin{tabular}{|c|c|c|c|c|c|c|}
\hline Variables & Total & $M$ & $S$ & D.S & I. $C$ & \\
\hline & \multicolumn{6}{|l|}{ Cohesión } \\
\hline & Calidad de trabajo (ejecución) & 1685,0 & 76,59 & 189,30 & 13,7587 & $74,0-87,0$ \\
\hline & Atracción hacia el grupo & 1208,0 & 54,90 & 40,08 & 6,3314 & $53,0-59,0$ \\
\hline & Roles valorados & 4312,8 & 196,03 & 11513,7 & 107,30 & $169,1-188,8$ \\
\hline & Calidad de trabajo (interpersonales) & 413,0 & 18,77 & 43,70 & 6,611 & $14,0-24,0$ \\
\hline & \multicolumn{6}{|l|}{ Liderazgo } \\
\hline & Empatía & 715,0 & 32,50 & 2606,2 & 51,05 & $21,0-22,7$ \\
\hline & Orientación a la Tarea & 770,7 & 35,03 & 3195,79 & 56,53 & $21,6-24,0$ \\
\hline \multirow[t]{3}{*}{ Futbol Sala } & Influencia en la toma decisiones & 695,6 & 31,61 & 2253,68 & 47,47 & $20,0-22,3$ \\
\hline & Valores deportivos & 779,4 & 35,42 & 3234,91 & 56,87 & $22,7-24,0$ \\
\hline & Apoyo social & 650,7 & 29,57 & 2170,52 & 46,58 & $19,0-20,8$ \\
\hline
\end{tabular}

En cuanto a la variable liderazgo, la escala de mayor puntuación es Orientación a la tarea para fútbol $(\mu=24,47, \mathrm{DS}=$ 2.69) y voleibol $(\mu=23,73, \mathrm{DS}=3.15)$. Baloncesto $(\mu=22,21$, $\mathrm{DS}=2.54)$ y fútbol sala $(\mu=35,42, \mathrm{DS}=56.87)$ otorgan mayor puntuación a la escala de valores deportivos. Las puntuaciones bajas se obtienen en la escala de apoyo social para baloncesto $(\mu=18,10, \mathrm{DS}=2.26)$, fútbol $(\mu=18,82, \mathrm{DS}=$ $2.71)$, voleibol $(\mu=19,47, \mathrm{DS}=2.11)$ y fútbol sala $(\mu=29,57$, DS $=46.5$ ).

El análisis correlacional entre las variables de cohesión y li- derazgo con sus respectivas dimensiones, como se observa en la Tabla 3. El análisis indica que las dimensiones calidad de trabajo-aspectos relativos a la ejecución y valores deportivos tienen un índice de correlación positiva débil $(\mathrm{r}=.150)$. Estos resultados reflejan que la calidad de trabajo-aspectos relativos a la ejecución se asocia con los valores deportivos. Mientras que en las dimensiones calidad de trabajo-relaciones interpersonales e influencia en la toma de decisiones se obtuvo un índice de correlación no significativo $(\mathrm{r}=-.018)$, es decir, no existe relación entre estas dos dimensiones.

Tabla 3. Correlaciones de Pearson entre Cohesión y Liderazgo

\begin{tabular}{|c|c|c|c|c|}
\hline \multirow[b]{2}{*}{ Variables } & \multicolumn{4}{|c|}{ Cohesión } \\
\hline & $\begin{array}{c}\text { Calidad de trabajo } \\
\text { (ejecución) }\end{array}$ & Atracción hacia el grupo & Roles valorados & $\begin{array}{l}\text { Calidad de trabajo } \\
\text { (interpersonales) }\end{array}$ \\
\hline \multicolumn{5}{|l|}{ Liderazgo } \\
\hline Empatía & $.041^{*}$ & $-.006^{*}$ & $-.012^{*}$ & $-.066^{*}$ \\
\hline Orientación a la tarea & $.115^{*}$ & $.044^{*}$ & $.064^{*}$ & $.049^{*}$ \\
\hline $\begin{array}{l}\text { Influencia en la toma } \\
\text { decisiones }\end{array}$ & $-.018^{*}$ & $-.037^{*}$ & $-.004^{*}$ & $-.045^{*}$ \\
\hline Valores deportivos & $.150^{*}$ & $.019^{*}$ & $.065^{*}$ & $-.016^{*}$ \\
\hline Apoyo social & $.122^{*}$ & $-.066^{*}$ & $.008^{*}$ & $-.056^{*}$ \\
\hline
\end{tabular}

${ }^{*}$ la correlación es significativa a nivel de 0.05$) \mathrm{P} \leq .05$

Entre las dimensiones atracción hacia el grupo y orientación a la tarea, se da una correlación positiva muy débil casi nula $(r=.044)$. En la dimensión atracción hacia el grupo y empatía, el índice de correlación negativa es muy débil ( $\mathrm{r}=$ -.006). Con respecto al índice de correlación de la dimensión roles valorados y valores deportivos $(r=.065)$, se da una correlación muy débil.
Para voleibol, como se observa en la Tabla 4, las dimensiones que se relacionan en mayor medida corresponden a calidad de trabajo-aspectos relativos a la ejecución con orientación a la tarea $(r=.227)$ siendo una correlación positiva moderada. La dimensión calidad de trabajo-relaciones interpersonales se correlaciona negativamente con la dimensión Orientación a la tarea $(r=-.005)$, es decir hay una relación nula. 
Tabla 4. Correlaciones de Pearson entre las dimensiones Cohesión y Liderazgo en voleibol.

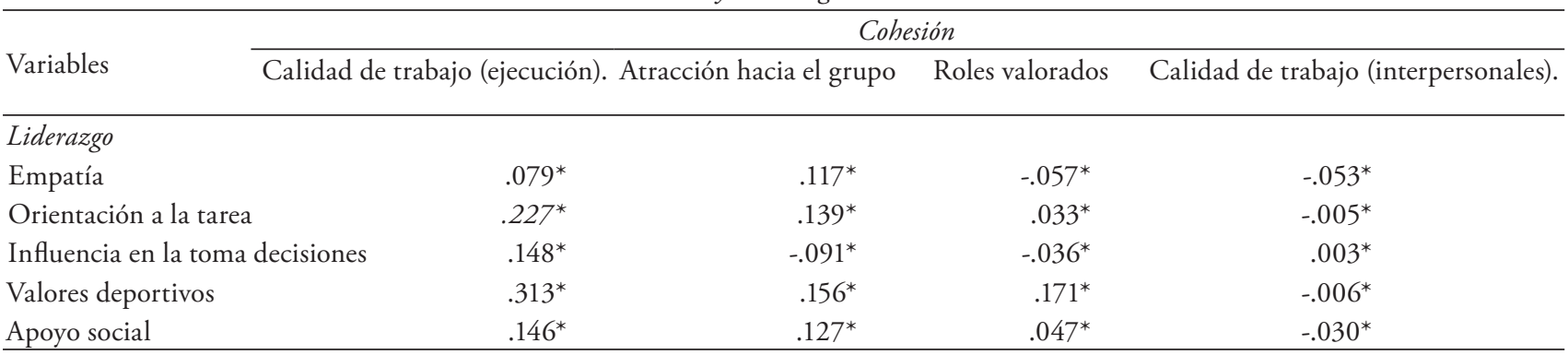

(* la correlación es significativa a nivel de 0.05$) \mathrm{P} \leq .05$

De acuerdo a los datos de la Tabla 5, las dimensiones que se relacionan de forma positiva y moderada en Baloncesto, son calidad de trabajo-aspectos relativos a la ejecución y em- patía $(\mathrm{r}=.230)$. La dimensión atracción hacia el grupo y valores deportivos se correlacionan negativamente $(\mathrm{r}=-.022, \mathrm{P}$ $\leq .05)$, es decir hay una relación nula.

Tabla 5. Correlaciones de Pearson entre las Cohesión y Liderazgo en baloncesto.

\begin{tabular}{|c|c|c|c|c|}
\hline \multirow[b]{2}{*}{ Variables } & \multicolumn{4}{|c|}{ Cohesión } \\
\hline & $\begin{array}{c}\text { Calidad de trabajo } \\
\text { (ejecución). }\end{array}$ & Atracción hacia el grupo & Roles valorados & $\begin{array}{l}\text { Calidad de trabajo } \\
\text { (interpersonales). }\end{array}$ \\
\hline \multicolumn{5}{|l|}{ Liderazgo } \\
\hline Empatía & $.230^{*}$ & $.137^{*}$ & $.053^{*}$ & $-.437^{*}$ \\
\hline Orientación a la tarea & $.072^{*}$ & $.113^{*}$ & $-.108^{*}$ & $-.065^{*}$ \\
\hline $\begin{array}{l}\text { Influencia en la toma } \\
\text { decisiones }\end{array}$ & $.077^{*}$ & $.098^{*}$ & $-.039 *$ & $.024^{*}$ \\
\hline Valores deportivos & $.005^{*}$ & $-.022^{*}$ & $-.116^{*}$ & $-.217^{*}$ \\
\hline Apoyo social & $.154^{*}$ & $-.037^{*}$ & $-.130^{*}$ & $-.325^{*}$ \\
\hline
\end{tabular}

$\left({ }^{*}\right.$ La correlación es significativa a nivel de 0.05$) \mathrm{P} \leq .05$

La siguiente tabla, muestra que para fútbol las dimensiones calidad de trabajo-aspectos relativos a la ejecución y orientación a la tarea, presentan una correlación positiva moderada $(r=.243)$, es decir, que al aumentar la orientación a la tarea, la calidad de trabajo aumenta (Ver Tabla 6). Así mismo se observa que las dimensiones Atracción hacia el grupo y Apoyo social presentan una correlación negativa nula $(\mathrm{r}=-.035)$.

Tabla 6. Correlaciones de Pearson entre las dimensiones Cohesión y Liderazgo en fútbol.

\begin{tabular}{lcccc}
\hline \multirow{2}{*}{ Variables } & \multicolumn{3}{c}{ Cohesión } \\
\cline { 2 - 5 } & $\begin{array}{c}\text { Calidad de trabajo } \\
\text { (ejecución). }\end{array}$ & $\begin{array}{c}\text { Atracción hacia el } \\
\text { grupo }\end{array}$ & Roles valorados & $\begin{array}{c}\text { Calidad de trabajo } \\
\text { (interpersonales). }\end{array}$ \\
\hline Liderazgo & $-.093^{*}$ & $-.062^{*}$ & $-.144^{*}$ & $-.061^{*}$ \\
Empatía & $.243^{*}$ & $.219^{*}$ & $.106^{*}$ & $-.066^{*}$ \\
Orientación a la tarea & $-.050^{*}$ & $.020^{*}$ & $-.064^{*}$ & $-.117^{*}$ \\
Influencia en la toma decisiones & $-.061^{*}$ & $.037^{*}$ & $-.149^{*}$ & $-.061^{*}$ \\
Valores deportivos & $-.099^{*}$ & $-.035^{*}$ & $-.109^{*}$ & $-.067^{*}$ \\
Apoyo social & & &
\end{tabular}

$\left({ }^{*}\right.$ La correlación es significativa a nivel de 0.05$) \mathrm{P} \leq .05$

Para Fútbol sala, las dimensiones atracción hacia el grupo y empatía tienen un índice de correlación positiva débil $(\mathrm{r}=.111)$ al igual que las dimensiones atracción hacia el grupo $\mathrm{y}$ valores deportivos $(\mathrm{r}=.111)$. En cuanto a las dimensiones calidad de trabajo-relaciones interpersonales y toma de decisiones $(r=-.085)$, su relación es nula ya que poseen una correlación negativa baja, tal y como se observa en la Tabla 7. 
Tabla 7. Correlaciones de Pearson entre las dimensiones Cohesión y Liderazgo en fútbol sala.

\begin{tabular}{|c|c|c|c|c|}
\hline \multirow[b]{2}{*}{ Variables } & \multicolumn{2}{|c|}{ Cohesión } & \multirow[b]{2}{*}{ Roles valorados } & \multirow[b]{2}{*}{$\begin{array}{c}\text { Calidad de trabajo } \\
\text { (interpersonales) }\end{array}$} \\
\hline & $\begin{array}{c}\text { Calidad de trabajo } \\
\text { (ejecución) }\end{array}$ & $\begin{array}{c}\text { Atracción hacia el } \\
\text { grupo }\end{array}$ & & \\
\hline \multicolumn{5}{|l|}{ Liderazgo } \\
\hline Empatía & $.100^{*}$ & $.111^{*}$ & $-.181^{*}$ & $-.090^{*}$ \\
\hline Orientación a la tarea & $.103^{*}$ & $.108^{*}$ & $-.176^{*}$ & $-.096^{*}$ \\
\hline Influencia en la toma decisiones & $.095^{*}$ & $.110^{*}$ & $-.183^{*}$ & $-.085^{*}$ \\
\hline Valores deportivos & $.102^{*}$ & $.111^{*}$ & $-.180^{*}$ & $-.096^{*}$ \\
\hline Apoyo social & $.094^{*}$ & $.108^{*}$ & $-.189^{*}$ & $-.097^{*}$ \\
\hline
\end{tabular}

$\left({ }^{*}\right.$ La correlación es significativa a nivel de 0.05$) \mathrm{P} \leq .05$

\section{Discusion}

En cuanto a los resultados derivados de este estudio, cuyo propósito inicial fue determinar la relación existente entre la cohesión y el liderazgo de los equipos deportivos adscritos a Indeportes Boyacá; se determinó realizar una validación de contenido para los instrumentos, mostrando índices altos para los criterios de pertinencia (0.994), suficiencia (0.999) y ubicación (0.999) para la EELD; y para el IMCODE en los criterios de fiabilidad (1), suficiencia (0.999) y ubicación (0977); dando como resultado que estos instrumentos poseen muy buena fiabilidad en concordancia entre los observadores (Validación de instrumentos, 2009). Sin embargo, para el criterio claridad se obtuvieron puntuaciones bajas (EELD 0.3456; IMCODE 0.038), revelando la necesidad de realizar ajustes a los instrumentos.

Por otra parte, la validez de criterio muestra un Alpha crombach muy alto para EELD (0.98) y alto para IMCODE (0.779), siendo estas muy significativas, lo que indica una correlación positiva alta mostrando fiabilidad de los instrumentos; es decir, cuanto más heterogéneo es el grupo de deportistas mayor es el coeficiente de confiabilidad.

Los resultados de los análisis descriptivos con respecto a la media, indican que con relación a la variable cohesión; la dimensión que logro una mayor puntuación fue calidad de trabajo-aspectos relativos a la ejecución $(\mu=74,54$; DS= 10.6); por otro lado la dimensión calidad de trabajo-relaciones interpersonales obtuvo la menor puntuación $\quad(\mu=18,73$; $\mathrm{DS}=5.66$ ); indicando que posiblemente, los deportistas de los equipos objeto de estudio tienen una inclinación hacia una cohesión dirigida a la tarea. A partir de la cual se refleja el grado en que los miembros del grupo trabajan juntos para alcanzar objetivos comunes, teniendo en cuenta los roles asignados con el fin de obtener un rendimiento que el grupo considere como exitoso (Carron, et al. 1985; Carron, 1998 citados por Leo, et al. 2008).

Por otra parte, se evidencia, que en la variable liderazgo la escala que reporto una mayor calificación fue orientación a la tarea $(\mu=23,50, D S=3.17)$ y apoyo social obtuvo una baja calificación $(\mu=18,92, \mathrm{DS}=2.44)$; esto muestra que en las ligas deportivas del departamento de Boyacá - Colombia, los lideres se encuentran orientados a la tarea, es decir, que existe en los mismos una tendencia a trabajar enfocándose en la consecución de los objetivos planteados con el fin de buscar el máximo rendimiento y productividad, dejando en un segundo plano las relaciones sociales o interpersonales (Fiedler, 1967 citado por Dosil, 2004).

Contrario a lo expuesto anteriormente, fútbol sala otorga una mayor puntuación a la dimensión roles valorados $(\mu=$ 196,03; DS= 107.30), indicando que dentro de este equipo existe una percepción clara acerca de los roles que cada deportista desempeńa, siendo cada uno de estos aceptados por el resto de los miembros, este equipo otorga mayor importancia a la cohesión social, en la que existe una motivación para desarrollar y mantener las relaciones sociales dentro del equipo, de modo que los miembros perciban que existe un clima recíproco de apoyo y de seguridad, en el que todos participan expresando opiniones e ideas, aumentando así la cohesión social (Carless y De Paola, 2000 citados por Picazo, et al., 2009).

En cuanto a la variable liderazgo, la escala que obtuvo una mayor puntuación fue valores deportivos $(\mu=35,42$; DS= 56.87); lo que indica una predominancia de líderes orientados a las relaciones sociales los cuales dan más importancia al manejo de aspectos comunicativos del equipo y las relaciones interpersonales intentando, buscar en lo posible un equilibrio entre asumir su rol y sentirse bien con el (Fiedler, 1967 citado por Dosil, 2004). De este modo el líder procura fomentar un buen clima grupal, siendo un referente para los compañeros, ya que encamina sus acciones hacia el bienestar de los miembros del equipo.

Con respecto al análisis general de correlación, las dimensiones que indican una relación moderada son calidad de trabajo-aspectos relativos a la ejecución y valores deportivos $(\mathrm{r}=.150)$; esto implica que en cierta medida, los deportistas aprecian la importancia que tiene el trabajo en equipo en la consecución del éxito deportivo teniendo en cuenta los roles 
asignados dentro del mismo (García, et al., 2006), el fomento de un buen clima grupal y la preocupación por el bienestar de sus miembros (Arce, et al., 2008). De este modo, se puede mencionar que teniendo en cuenta la dimensión calidad de trabajo-aspectos relativos a la ejecución, posiblemente los deportistas se enfoquen hacia una cohesión orientada a la tarea, situación que concuerda con los hallazgos de los estudios realizados por Carron, et al. (1985 citados por Leo, et al., 2008).

En cuanto a la dimensión valores deportivos, se muestra que los deportistas de esta investigación se dirigen hacia un liderazgo orientado a los aspectos sociales, esto apoyado por los estudios realizados por Fiedler (1967, citado por Dosil, 2004) en los cuales hace una diferenciación entre los lideres orientados a la relación y a la tarea; siendo los primeros, los que inciden en aspectos comunicativos del grupo y relaciones interpersonales buscando siempre un equilibrio en asumir el papel y sentirse cómodo con él. Es importante anotar, que la efectividad de cada uno de estos estilos de liderazgo, depende básicamente de tres factores situacionales: a) las relaciones entre los integrantes del equipo y el líder, b) la estructura jerárquica de la actividad deportiva y c) la posición que asuma el líder (de mando, autoridad, permisividad, etc.).

Al realizar el análisis de correlación de Pearson por deportes, se observa, que el equipo de baloncesto relaciona en mayor medida las dimensiones calidad de trabajo-aspectos relativos a la ejecución con empatía $(\mathrm{r}=.230)$; manteniendo una cohesión enfocada en la tarea y un liderazgo enfocado a las relaciones sociales. En voleibol, las dimensiones que se relacionan son, calidad de trabajo-aspectos relativos a la ejecución y orientación a la tarea $(\mathrm{r}=.227)$, y en fútbol las dimensiones calidad de trabajo-aspectos relativos a la ejecución y orientación a la tarea $(r=.243)$, centrándose estos dos grupos tanto en una cohesión, como en un liderazgo dirigidos a la tarea, referida a la actitud hacia el rendimiento, tanto en entrenamientos como en competición y a la forma de afrontar el juego (Arce, et al., 2008).

Es importante señalar, como indica C. T. García, (2004) es primordial que en el equipo existan buenas relaciones entre todos los miembros que lo componen, ya que esto permite mayores posibilidades de conseguir éxito en las competiciones y dispone mayores recursos y posibilidades para afrontar cualquier obstáculo que se presente durante la competencia; sin embargo, algunos equipos han conseguido un gran rendimiento deportivo sin poseer buenas relaciones entre jugadores o relaciones entre éstos y el entrenador; situación que posiblemente sea el resultado del establecimiento de objetivos por cada uno de los integrantes del equipo, mediante los cuales se busca el reconocimiento individual o sobresalir dentro del grupo sin que exista un objetivo común claro hacia el cual se dirijan todos los deportistas miembros del mismo.

A diferencia de los demás equipos, los deportistas de fútbol sala se orientan en mayor medida hacia una cohesión social (Carless y De Paola, 2000 citados por Picazo, et al., 2009). En cuanto al liderazgo deportivo, se manteniene una orientación social debido a que como lo expresa Dosil, (2004) características como aquellas que identifican al equipo desde el punto de vista psicológico; condiciones ambientales, de juego e interacciones entre los miembros del equipo hacen que se requiera de continuos procesos de comunicación, cooperación, confianza y compromiso entre los miembros; así mismo, es necesario que dentro de éste se establezcan una serie de normas que fomenten un buen clima grupal que favorezcan la creación de estrategias que permitan seguir las instrucciones del líder.

Los resultados de esta investigación, reflejan la importancia de estudiar diferentes dinámicas grupales, que ayuden a comprender tanto el comportamiento individual como social de los grupos deportivos, ya que esto permitirá realizar posteriores intervenciones que procuren incentivar el trabajo en el fortalecimiento y mantenimiento de los diferentes componentes de un equipo, para así reforzar el rendimiento deportivo.

De aquí, que sea de vital importancia, tal y como lo expresan R. Paglilla y D. Paglilla, (2005) tener en cuenta que la dinámica interna de los integrantes del equipo debe ser conducida de una manera acertada, de modo que esta refleje una ventaja en la consecución de los objetivos grupales evitando el fracaso por falta de compromiso; llegando de este modo a la optimización del rendimiento deportivo. De acuerdo con lo anterior, en un inicio, la intervención psicológica con grupos deportivos deberá enfocarse, como lo señalan Carron y Brawley, (2000, citados por Picazo et al., 2009), en fomentar una cohesión dirigida hacia la tarea donde el establecimiento de objetivos y normas se perciban como factores determinantes en el fortalecimiento y mantenimiento del equipo, superada esta etapa se trabaja en el fomento de una cohesión dirigida a las relaciones sociales con el fin de terminar fortaleciendo el equipo, tanto en aspectos relacionados a la tarea como en aspectos sociales.

Así mismo, para garantizar el desarrollo de la cohesión dentro de los equipos deportivos, es importante tener en cuenta los factores ambientales, personales, de liderazgo y de equipo propuestos por Carron (1982, citado por Velásquez, 2005) en su modelo de cohesión; los cuales pueden llegar a afectar el rendimiento del equipo al no ser manejados o controlados de manera efectiva, dado que la intervención oportuna de estos factores dirige significativamente la cohesión hacia la unión por actividades o tareas que se relacionen con el rendimiento de los objetivos propuestos por el equipo.

Con relación al liderazgo deportivo, estos resultados muestran la importancia de mantener en los equipos, líderes orientados a las relaciones sociales dado que sus características de disfrute de las relaciones sociales, la generosidad en la valoración y relación con los demás, entusiasmo, creatividad, 
organización, compromiso, etc. Según Davies (1991, citado por Ruiz, 2006), contribuyen en gran medida a lograr un equilibrio dentro del equipo, dado que las características del entrenador generalmente cumplen con las particularidades de un líder orientado a la tarea, mientras que el deportista se orienta en gran medida por un liderazgo orientado a las relaciones sociales. Es así, como los estudios realizados por Loughead y Hardy (2005, citados por Arce et al. 2008), indican que mientras los entrenadores presentan comportamientos autoritarios y orientados a la tarea, los jugadores líderes tienden a mostrar comportamientos de apoyo social orientados a las relaciones, obteniendo en el campo de juego mayor aceptación por parte de sus compañeros, evidenciando así un equilibrio con relación al liderazgo.

Finalmente, se puede concluir que el desarrollo de la cohe- sión y el liderazgo deportivo en las ligas de baloncesto, fútbol, fútbol sala y voleibol de Indeportes Boyacá, logran mantener una proporción adecuada, debido a que al estar la cohesión dirigida a la tarea y el liderazgo a las relaciones sociales, los integrantes de estas ligas posiblemente pueden encontrar en sus equipos una serie de satisfactores tanto a nivel personal como social.

Esta investigación se constituye en un valioso aporte y acercamiento a la medición y entendimiento de la cohesión y liderazgo en equipos deportivos de Colombia, específicamente los del Departamento de Boyacá y se considera un intento inicial por realizar investigación que contribuya al avance científico y en procura de intervenir como psicólogos del deporte basados en la evidencia y aplicabilidad.

\section{Referencias}

Arce, C., Torrado, J., Andrade, E., Garrido, J. y De Francisco, C. (2008). Elaboración de una escala para la medida de la capacidad de liderazgo de los deportistas de equipo. Psicothema, 20, 913-917. Recuperado en Julio 13, 2009, disponible en http://www.psicothema.com.

Briones, E. y Tabernero, C. (2005). Formación cooperativa en grupos hetereogéneos. Psicothema, 3, 396-403. Recuperado en Junio 24, 2009, disponible en http://www.psicothema.com.

Díez, A. y Márquez, S. (2005). Utilización de sociogramas para la valoración de la cohesión interna de los jugadores de un club de fútbol. European Journal of Human Movement, 14, 37-52. Recuperado en Junio 22, 2009, disponible en http://www. dialnet.unirioja.es. Diferencia Equipo-Grupo. (2009). Recuperado en Abril 15, 2010, disponible en http:// cordobasket.blogspot.com/2009/12/diferencia-equipo-grupo.html.

Dosil, J. (2004). Psicología de la actividad física y del deporte. Madrid, España: Mc Graw Hill.

García, C.T. (2004). Intervención para mejorar la cohesión de equipo y el establecimiento de roles de liderazgo entre los jugadores: El trabajo de aspectos psicológicos en fútbol a través del entrenamiento integrado. CV Ciencias del Deporte, 9-25. Recuperado en Julio 11, 2009, disponible en http://www.dialnet.unirioja.es

García-Mas, A. (2002). Funcionamiento de los equipos deportivos: dinámica externa e interna y liderazgo deportivo. Manual de Psicología del Deporte, 204 - 235. Recuperado en Septiembre 2, 2009, disponible en http://www.dialnet.unirioja.es.

García-Mas, A., Olmedilla, A., Morilla, M., Rivas, C., García, O. y Ortega, E. (2006). Un nuevo modelo de cooperación deportiva y su evaluación mediante un cuestionario. Psicothema, 18, 425-432. Recuperado en Julio 13, 2009, disponible en http://www.psicothema.com.

García, E. M., Rodríguez, M., Andrade, E. M. y Arce, C. (2006). Adaptación del cuestionario MSCI para la medida de la cohesión en futbolistas jóvenes espańoles. Psicothema, 18, 668-672. Recuperado en Junio 25, 2009, disponible en http://www.psicothema.com.

González, O. (2007). Cohesión de equipo. Revista Digital, 12 (114). Recuperado en Julio 20, 2009, disponible en http://www.efdeportes.com.

Hardy, L. y Jones, G. (1996). Understanding Psychological Preparation for
Sport. Theory and Practice of Elite Performers. Chichester Wiley. Recuperado en Mayo 18, 2009, disponible en http://www.sportkinetics2007.com. Leo, F., García, T., Sánchez, P. y Parejo, I. (2008). Importancia de la percepción de eficacia para la mejora de la cohesión en el fútbol. Cuadernos de Psicología del Deporte. 8 (1), 47-60. Recuperado en Abril 18, 2009, disponible en http://www.psicodeporte.net.

Ortín, F. (2002). Dinámica y dirección de grupos deportivos. Manual de Psicología del Deporte, 145-163. Recuperado en Septiembre 2, 2009, disponible en http://www.dialnet.unirioja.es.

Paglilla, R. y Paglilla, D. (2005). La dinámica grupal de equipos deportivos. Planificación y conducción de equipos. Revista Digital, 10 (83). Recuperado en Octubre 20, 2009, disponible en http://www.efdeportes.com.

Picazo, C. Zornoza, A. y Peiró, J. (2009). Los procesos de participación social y participación orientada a la tarea y el aprendizaje como antecedentes de la cohesión grupal: Una perspectiva longitudinal. Psicothema, 21, 274-279. Recuperado en Julio 11, 2009, disponible en http://www. psicothema.com.

Ruiz, R. (2006). Diferencias de liderazgo en entrenadores de judo a nivel competitivo. Cuadernos de psicología del deporte. 6 (2), 21-38. Recuperado en Abril 27, 2009, disponible en http://www.psicodeporte.net.

Sabas, G. y Del Pino, M. (2009, Junio). Cohesión de grupo y su carácter predictor del rendimiento. Ponencia presentada en el primer encuentro on-line de psicología del deporte. Recuperado en Septiembre 25, 2009, disponible en http://sipd.files.wordpress.com/2009/06/ponencias_encuentro_online_sipd.pdf.

Salazar, W. (2006, Octubre). Avances en el estudio de la cohesión de equipo y su relación con el rendimiento. Ponencia presentada en el II congreso internacional y III congreso colombiano de psicología del deporte y el ejercicio físico, Bogotá, Colombia.

Validación de instrumentos. (2009). Recuperado en Abril 20, 2010, disponible en http://www.tesisperu.com/pastillas/28\%20Topico\%20\%20 Validacion\%20de\%20instrumentos.pdf.

Velásquez, M. (2005). Liderazgo y cohesión en grupos deportivos. Diálogos: discusiones en la psicología contemporánea, (2). Recuperado en Junio 27, 2009, disponible en http://www.digital.unal.edu.co 